\section{Disclaims Dishonesty.}

Indianapolis, Ind., April 14, 1899.

To the Editor:-In one of your "Minor Commente" of April 8 (vide JourNaL, p. 770), complaint is made concerning "one W. B. Clarke" (the "one" lest there might be two, perhaps), in a published communication "made up of alleged quatations from medical men, against vaccination," in which "isolated statements are quoted from noted physicians, but separated from their con text so as to make them express opinions contrary to the general tone of their published writings and their real sentiments," which presentation you characterize as "thoroughly dishonest" and characteristic of "unscrupulous cranks." Having long been a reader of the JournaL, the best medical journal in the world, and not wishing to fall under its condemnation, but desiring to be set right in the matter, please allow me to say that the article you refer to was mainly made up from the work "Smallpox and Vaccination," by Alex. M. Ross, M.A., F.R.S.L., Eng., of Montreal, who died a year ago, and who was very prominent in the profession. I have seen them in other works and pamphlets, but never before knew their honesty to be im pugned. When I used his quotations I had no doubt that they were bona-fide, and did not have their context to verify them by. There was, then, no dishonesty in their use. I can also furnish the source of all other quotations used, but will not now weary you with them. I will be perfectly satisfied to allow you to republish the article you criticise (if you have lost yours I can get you another), point out its unwarranted uses, and leave the matter to the judgment of your readers.

That the vaccination subject is a debatable one in its present shape (as are many other medical subjects), we see by the appearance of such laborious works as Crookshank's "History and Pathology of Vaccination," Creighton's "Jenner and Vaccination" and "Cowpox and Vaccinal Syphilis," and his article on "Vaccination" in the "Encyclopedia Britannica," White's "History of a Great Delusion," Pickering's "Sani. tation or Vaccination," and many emaller worke that could be mentioned. While $I$ believe in a scientific prophylaxis as $\mathrm{re}$ garde smallpox, inoculative in character, 1 do not intend to be dishonest or to use unfair means in discussing cowpox vaccina tion, nor do I need to. Yours respectfully,

W. B. Clarke, M.D.

[When an article quotes Niemeyer, Sir James Paget, Jonathan Hutchinson, F. H. Hamilton and others like them as opponents of vaccination, and uses statements applicable only to accidents from the old arm-to-arm method as against vaccination generally, one is excusable in questioning its fairness and candor, to state it mildly. But if these are all taken from the work of Dr. Ross, who is also quoted as declaring vaccination "an un witigated curse and the most destructive medical delusion which has ever afflicted the human race," the original dishon esty is traced a little farther back.-ED.]

\section{A Subliminal Miscarriage.}

RockFord, ILc., A pril 10, 1899.

To the Editor:--Probably more errors occur in sublimina argument through inductive conclusions, from the employ ment of indifferent or false data than is realized in our com mon philosophy; conclusions are drawn and made positive from simple parallels or seeming coincidences, and applied in theory as though actual facts; especially is this true where narrow ardor or impoverished avidity seeks to gratify a pretense, or fit a vacuum.

In no phase of philosophy is this truer than in the interminable maze of psychiatry where we deal with those attributes which make man kin to Deity and "only a little lower than the angels" ; where the border line between mediocrity and madness is as meager and tortuous as the tracks of the sea in a tempest; where Hahnemann was, and Eddy and
Dowie are now floundering; and the foam. and epray they raise is wrecking by its blinding veil weak minds that will not be piloted by trained thought into clear waters.

Only the shallow subliminal instances are given, that can be utilized for their conclusions and purposes; the abortive or negative ones are ignored by the fanatic and seldom chronicled by the thoughtful and candid, and thus a store of valuable data is lost to actual science and only the vague side of the subliminal sphere brought to view.

To perform a duty on right lines this instance is.given :

During the recent winter a Mrs. $R$., an American, aged 28 years, the mother of three children, became the subject of a pelvic abscess necessitating her removal from her home to the local hospital, for an operation; with many.others of her rank the thought of going to a hospital was much dreaded by herself and bewailed by her family until it was their subject of talk by day and evidently their dreams by night; for, pending the argument and consent of all concerned, the subject herself, her mother-in-law, her maid, her foster-mother and hus. band, all, on either the same or successive nights dreamed seeing their loved one carried away in the city ambulance; had been present at her death, had witnessed her burial robes, flowers, casket, and interment, with a uniformity that was startling in detail even to real consciousness when in turn they were graphically recited. Notwithstanding this quintuple subliminal testimony, the patient passed through the opera tion, rallied and recovered without a single untoward event, returning to her home on the eleventh day, restored and happy.

Then all in timid and penitent turn related their dreamy observations of a fortnight before, with such graphic and appropriate embellishment as their station, imagination and command of words permitted, without one being able to add the ghoulish, "I told you so," to the finalé. Spiritualiste will find little to tempt them in this episode and the psychist may record it as an object for future reference as a rare negative narrative in his search for truth. D. Lichty, M.D.

\section{For Whose Benefit Do We Write?}

Fargo, N. D., April 11, 1899.

To the Editor:-Apropos of Dr. Howle's letter in the JovRNAL (vide April 8, p. 777) perhape I may be able to throw a little side light on the subject.

Recently the representative of a drug house in the East came into my office, and picking up a reprint of an article of mine that had just appeared in a prominent journal, eaid : "Say, Doctor, if you will write an article recommending the use of our prepara tion for that class of work, I will reward you well." Here was an inducement to help out the manufacturer and at the same time look out for number one. While the preparation he spoke of is quite a good one, there are any number of just such preparations put up by different houses, and most of them just as good as his. It is needless to say the bribe was not accepted. I do not mean to say that all such articles are "bought," as he wished to buy mine, but some of them evidently are, if not in one way then in another. It also frequently serves to bring one's name before the profession, although this object is of ten miseed. I know that when such "reprints" and "extracts" come to my desk they are more apt to go into the waste basket than to be read, digested and remembered, and I am not alone in thus dealing with them. The author should write for the benefit of his readers and not for that of the manufacturer.

W. L. Grant, M.D.

South McAlester, I. T., April 12, 1899.

To the Editor:-I note a communication in the Journar (vide April 8, p. 777), anent the laudation of nostrums by the profession. The writer intimates that there is a Senegambian in the fuel-stack, but seems unable to locate his whereabouts. I think I have found this swart individual. An article of 